\title{
BreastCore
}

\section{ABC4 Consensus: Assessment by a German Group of Experts}

\author{
Nadia Harbeck ${ }^{a}$ Diana Lüftner ${ }^{b} \quad$ Norbert Marschner $^{c} \quad$ Michael Untch $^{d}$ Doris Augustin ${ }^{\mathrm{C}}$ \\ Susanne Briest ${ }^{f}$ Johannes Ettl ${ }^{g}$ Renate Haidinger ${ }^{h}$ Lothar Müller ${ }^{i}$ Volkmar Müller ${ }^{j}$ \\ Eugen Ruckhäberle $^{k}$ Rachel Wuerstlein ${ }^{a}$ Christoph Thomssen'
}

\footnotetext{
a Breast Center and CCC Munich, University of Munich (LMU), Department of Obstetrics and Gynecology, Munich, Germany;

b Medical Department of Hematology, Oncology and Tumor Immunology, Humboldt University Berlin, Charité Campus Benjamin Franklin, Berlin, Germany;

c Joint Practice for Interdisciplinary Oncology and Hematology, Freiburg i.Br., Germany;

${ }^{d}$ Gynecology Clinic, HELIOS Hospital Berlin-Buch, Berlin, Germany;

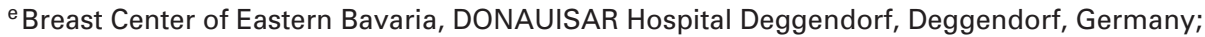

fUniversity Gynecological Hospital, Leipzig, Germany;

gWomen's Hospital and Polyclinic for Gynecology and Obstetrics, Klinikum Rechts der Isar Hospital, Munich, Germany;

h Brustkrebs Deutschland e.V., Hohenbrunn, Germany;

iPractice Focusing on Oncology Leer-Emden-Papenburg, Leer, Germany;

iUniversity Women's Hospital Hamburg-Eppendorf, Hamburg, Germany;

kWomen's Hospital, University Hospital Düsseldorf, Düsseldorf, Germany;

'University Hospital and Polyclinic of Gynecology, Martin Luther University, Halle (Saale), Germany
}

\section{Keywords}

ABC4 $\cdot$ Advanced breast cancer .

HR-positive/HER2-positive $\cdot$ BRCA-associated .

Brain metastases - Personalized medicine

\section{Summary}

The Advanced Breast Cancer Fourth Consensus (ABC4) on diagnosis and treatment of advanced breast cancer (ABC) again took place in Lisbon, on November 2-4, 2017, and was chaired by Fatima Cardoso, MD, PhD. This year's contents focused very much on new developments in the treatment of $A B C$. For example, the significance of inhibition of cyclin-dependent kinases 4 and 6 (CDK4/6) in hormone receptor (HR)-positive $A B C$, of dual antibody blockade in human epidermal growth factor re-

Nadia Harbeck, Diana Lüftner, Norbert Marschner, Michael Untch, Rachel Wuerstlein, and Christoph Thomssen were members of the writing committee. Nadia Harbeck and Christoph Thomssen were ABC panel members. Nadia Harbeck was an ABC Scientific Committee member. Renate Haidinger was a member of the Faculty of ABC4.

The comments by the German expert group are based on the voting results by the $\mathrm{ABC} 4$ panelists on site in Lisbon on Saturday, November 4, 2017. The present manuscript is an opinion from the perspective of the participating German breast cancer experts. The official ABC4 consensus will be published in The Breast under the authorship of the ABC4 panelists. ceptor 2 (HER2)-positive ABC, and of poly(ADP-ribose) polymerase (PARP) inhibition in triple-negative $A B C$, as well as the potential therapeutic consequences, were discussed. Other key issues were BRCA-associated breast cancer, treatment of brain metastases, and personalized therapy decision-making using molecular testing (socalled 'precision medicine'). As in past years, an important objective of the $A B C$ conference was cooperation with representatives of patient organizations from around the world. This cooperation was further intensified during the $A B C 4$. Following the main conference, the 'Global Alliance' was founded, with the goal of publicizing and coordinating measures necessary worldwide from the patient advocates' standpoint. - The ABC consensus inevitably cannot accommodate country-specific needs, due to the truly global expert panel. Therefore, a working group of German breast cancer experts commented - as in the past years - on the on-site voting results by the $A B C$ panelists upon which the final $A B C 4$ consensus will be based, with particular consideration of the German guidelines on diagnosis and treatment of breast cancer for everyday treatment in Germany.

(c) 2018 S. Karger GmbH, Freiburg

\section{KARGER}

(c) 2018 S. Karger GmbH, Freiburg 


\section{Introduction}

The international Advanced Breast Cancer (ABC) consensus conference on diagnosis and treatment of advanced breast cancer takes place every 2 years. The goal of the $\mathrm{ABC}$ consensus is to internationally harmonize and standardize the treatment situation of patients with locally advanced or metastatic breast cancer. This year, on November 2-4, 2017, the international experts met for the fourth time since 2011. As always, the ABC conference took place in Lisbon.

The 2017 interdisciplinary $\mathrm{ABC}$ panel (table 1) consisted of 42 internationally renowned breast cancer experts, including 5 patient advocates and 1 oncology nurse. 2 breast cancer experts from Germany, Prof. Nadia Harbeck, Munich, and Prof. Christoph Thomssen, Halle/Saale, were part of the ABC4 panel. Prof. Harbeck was also a member of the 3-person 'Scientific Committee' of the $\mathrm{ABC} 4$ conference. For the first time, Renate Haidinger (Brustkrebs Deutschland e.V.) was part of the 'Faculty' as a patient advocate.

In the focus of this year's ABC4 consensus were new developments in the treatment of advanced and metastatic breast cancer. New substance groups, such as, e.g., cyclin-dependent kinases 4 and $6(\mathrm{CDK} 4 / 6)$ inhibitors, and the impact of recent evidence on the respective therapeutic algorithms were discussed. The treatment options for patients with $B R C A$-associated breast cancer or with brain metastases were discussed intensively. Emphasis was also put on precision medicine with its potential to further individualize therapy decision-making by molecular testing. Cooperation with representatives of patient organizations from around the world was again an important objective of the $\mathrm{ABC}$ conference and was thus further intensified.

The ABC conference was organized by the European School of Oncology (ESO), in cooperation with the European Society of Clinical Oncology (ESMO). As in the past years, the recommendations by the ABC4 consensus 2017 will be published in the peer-reviewed journal The Breast [1-3], in cooperation with different international expert associations and patient organizations, among others.

The objective of the $\mathrm{ABC}$ conference is to create the basis for individualized, evidence-based therapy decisions in $\mathrm{ABC}$. On site, the statements were voted on by the panelists with 'yes' (approve), 'no' (reject), or 'abstain'. For the first time, a fourth voting option was introduced this year for some questions, namely 'insufficient data'. It was an option if a panel member thought that there were insufficient data to vote 'yes' or 'no'. In addition, a new evidence grading system based on the ESMO therapy guidelines [4] (fig. 1) was introduced.

In order to prevent redundancies, only new or modified questions were voted on during the $\mathrm{ABC} 4$. Those statements of the previous conferences $\mathrm{ABC} 1-3$ that were not voted on again continue to be valid. The ABC4 panelists were asked to vote based on evidence from clinical trials and to disregard whether a particular therapy option is available in every country or not. The objective is to help provide medically necessary treatments in these countries as well. Health political questions were not and will not be discussed in the consensus statements.

The rationale of the present publication is to comment on the on-site $\mathrm{ABC} 4$ voting results from the German perspective [5-7]
Table 1. $\mathrm{ABC} 4$

consensus panelists

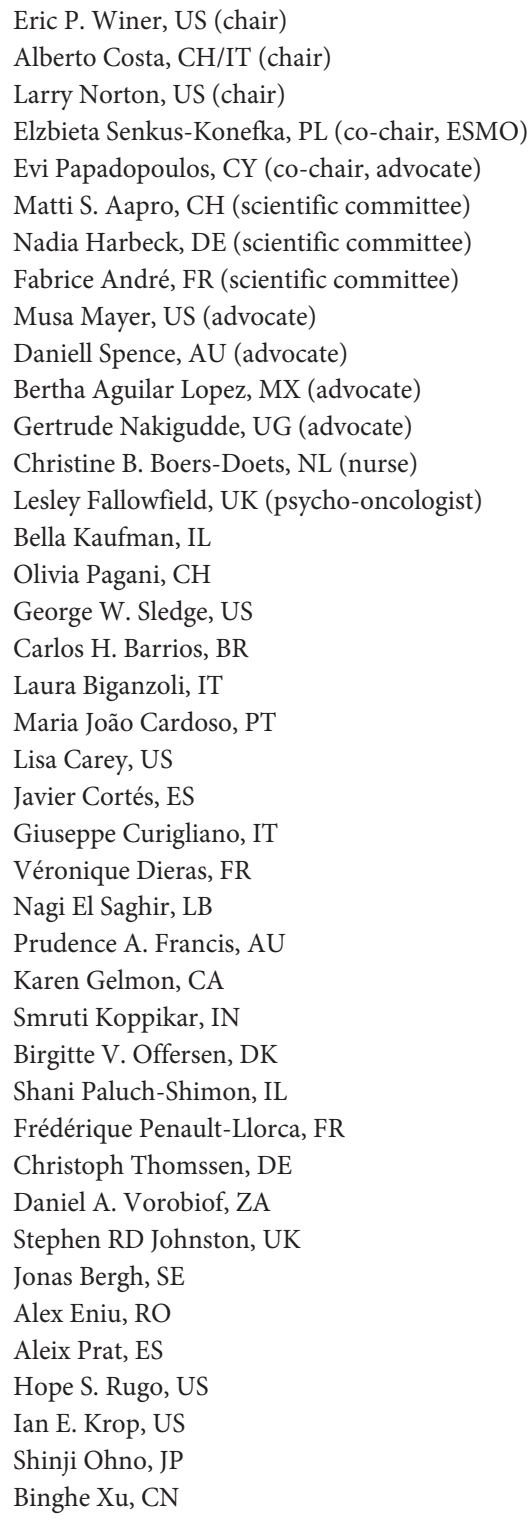

and to discuss them regarding their impact on the treatment routine in Germany. This seems necessary since the voting results are based on the opinions of experts in different fields and because country-specific viewpoints that may not apply to the German situation cannot be ruled out. The German experts focus on the voting results on site. Therefore, subsequent modifications made by the $\mathrm{ABC} 4$ panel for the upcoming consensus publication cannot be taken into consideration for this manuscript.

\section{HR-Positive, HER2-Negative Advanced or Metastatic Breast Cancer}

\section{Ovarian Function Suppression or Ablation}

Premenopausal patients with hormone receptor-positive $(\mathrm{HR}+)$ /human epidermal growth factor receptor 2-negative (HER2-) advanced or metastatic breast cancer have rarely been 


\section{New grading system for ESMO Guidelines}

LEVELS OF EVIDENCE

\begin{tabular}{|l|l|}
\hline I & $\begin{array}{l}\text { Evidence from at least one large randomized, controlled trial of good methodological } \\
\text { quality (low potential for bias) or meta-analyses of well-conducted randomized trials } \\
\text { without heterogeneity. }\end{array}$ \\
\hline II & $\begin{array}{l}\text { Small randomizcd trials or largc randomized trials with a suspicion of bias (lowcr } \\
\text { methodological quality) or meta-analyses of such trials or of trials with demonstrated } \\
\text { heterogeneity. }\end{array}$ \\
\hline III & Prospective cohort studies. \\
\hline IV & Retrospective cohort studies or case-control studies. \\
\hline V & Studies without control group, case reports, experts' opinions. \\
\hline
\end{tabular}

\section{GRADES OF RECOMMENDATION}

Fig. 1. Level-of-evidence grading system used by the $\mathrm{ABC} 4$ panelists. Adapted with permission from the Infectious Diseases Society of America United States Public Health Service Grading System [29].

\begin{tabular}{|l|l|}
\hline A & Strong evidence for efficacy with a substantial clinical benefit, strongly recommended. \\
\hline B & $\begin{array}{l}\text { Strong or moderate evidence for efficacy but with a limited clinical benefit, generally } \\
\text { recommended. }\end{array}$ \\
\hline C & $\begin{array}{l}\text { Insufficient evidence for efficacy or benefit does not outweigh the risk or the disadvantages } \\
\text { (adverse events, costs, ...), optional. }\end{array}$ \\
\hline D & Moderate evidence against efficacy or for adverse outcome, generally not recommended. \\
\hline E & Strong evidence against efficacy or for adverse outcome, never recommended. \\
\hline
\end{tabular}

randomized into endocrine therapy trials and, consequently, only limited trial data exist on the treatment of young patients. Therefore, a clear majority of the ABC4 panelists (94.5\%) emphasized that premenopausal patients require adequate ovarian function suppression or ablation (OFS) and should then be treated like postmenopausal patients. The ABC4 panel recommended treatment with an endocrine substance \pm applying targeted therapy. If premenopausal patients refuse OFS, treatment with tamoxifen is the only - but less effective - option (91.6\%). The panel (91.8\%) also stressed the need to design future trials evaluating new endocrinebased therapeutic strategies so that pre- and postmenopausal patients can be included (level of evidence (LoE): expert opinion). The German expert group agrees with all 3 statements and refers to the BCY3 consensus (Breast Cancer in Young Women) [8] supporting this as well.

\section{Use of Everolimus}

Patients with endocrine-resistant HR+/HER2- metastatic breast cancer can achieve a significantly longer median progression-free survival (PFS) if they receive the mammalian target of rapamycin (mTOR) inhibitor everolimus in addition to an aromatase inhibitor (AI) versus an AI alone. Even if an overall survival benefit was not shown, the combination of $\mathrm{AI}+$ everolimus is a valid therapeutic option for some patients, according to the majority vote (87.8\%; abstained: 9.7\%). However, the $\mathrm{ABC} 4$ panelists pointed out that the therapeutic decision must particularly consider potential side effects and the lack of a survival benefit (LoE: 1B). A majority (79.4\%; abstained: $15.3 \%$ ) of the ABC4 panelists accepted tamoxifen (LoE: 2B) and fulvestrant (LoE: expert opinion) as further combination partners of everolimus.
Everolimus in combination with an AI can also be an option for older patients. However, the ABC4 panelists (97.4\%) recommended consistent monitoring as well as proactive side effect management. They referred to the increased incidence of toxicities and even deaths in older patients in the everolimus-containing arm of the BOLERO-2 study $[9,10]$.

The German experts agree with all statements. They add that the combinations of fulvestrant + everolimus and tamoxifen + everolimus are not approved but nevertheless supported by evidence [11]. In addition to secondary prophylactic side effect management, primary prophylactic measures such as dexamethasone and other mouth washes are recommended by the German experts to avoid severe side effects.

\section{CDK4/6 Inhibition + AI}

A clear majority of the ABC4 panelists recommended first-line treatment with a CDK4/6 inhibitor in combination with an AI, which has a high level of evidence (1A) (89.7\%, abstain: 10.2\%). This recommendation only excludes patients who have disease progression within 12 months after adjuvant AI therapy. The $\mathrm{ABC} 4$ panelists based their vote on the significant median PFS advantage of about 10 months by adding a CDK4/6 inhibitor, in addition to an acceptable side effect profile [12-14]. The patient-reported quality of life was at least comparable to that under endocrine therapy alone in all quality-of-life analyses. There are no mature survival data yet. During the on-site discussion, the ABC4 panelists pointed out that it is important to make CDK4/6 inhibitors available in all countries. - The German experts agree with all statements. 


\section{CDK4/6 Inhibition + Fulvestrant}

The combination of CDK4/6 inhibitor + fulvestrant is a preferred option for patients with HR+/HER2- metastatic breast cancer who already received prior endocrine treatment and who did not receive a CDK4/6 inhibitor in the first line (LoE: $1 \mathrm{~A})$. This is valid regardless of the menopausal status. Pre-/perimenopausal patients additionally require OFS. The $\mathrm{ABC} 4$ panelists again assigned a high level of evidence (LoE: 1A) and justified their majority vote (90\%; abstained: 10\%) with the significant median PFS advantage of 6-7 months compared to endocrine therapy alone (fulvestrant) [15]. The ABC4 panelists pointed out that there is no mature overall survival data for this combination either.

The German experts agree: Targeted therapy should be added at the latest in the second line of endocrine-based therapy. From the German perspective, the median PFS is an important study endpoint from the patients' perspective. The quality-of-life analysis also shows better on-treatment quality of life for the combination with fulvestrant compared to the situation under endocrine therapy alone [16].

\section{Therapy Sequence after Endocrine-Based Therapy}

It is currently unclear what the optimal therapy sequence after endocrine-based first-line therapy looks like in HR+/HER2- metastatic breast cancer. Based on the majority vote $(94.7 \%$; abstain: $5.2 \%)$, therapy decisions need to take into account prior therapies in the (neo-)adjuvant setting or in the advanced setting, tumor burden, patient preferences, costs, and availability. However, the general statement by the $\mathrm{ABC} 4$ panelists that drug availability does not play a role in the consensus recommendations needs to be pointed out.

Based on the majority vote (73.6\%; abstained: $21.0 \%)$, the following therapeutic options are possible after endocrine-based first-line therapy for metastatic patients: tamoxifen, fulvestrant \pm CDK4/6 inhibitor or everolimus in combination with an AI, tamoxifen or fulvestrant. The ABC4 panelists also recommended megestrol acetate and estradiol as options for later lines of therapy. They emphasize, however, that there are currently no data from head-to-head studies that compare the various endocrinebased therapeutic options to one another or to monochemotherapy ( \pm bevacizumab). Results of ongoing trials must be awaited (LoE: $1 \mathrm{~A})$.

The German experts agree with this but suggest that there are currently no data on treatment beyond progression (TBP) with targeted drugs (CKD4/6 or mTOR inhibitors). They do not see an indication for high-dose progestins (e.g. megestrole acetate) or estradiol, also not in later lines of treatment (fig. 2). This is in line with the recommendations of the AGO (Arbeitsgemeinschaft Gynäkologische Onkologie, Working Group Gynecological Oncology) [5, 6].

Selection Criteria for Endocrine-Based Therapy Urgently Needed

Besides the HR status, there are currently no other validated predictive markers to identify those patients who would benefit from endocrine-based therapy with a CDK4/6 or mTOR inhibitor. Additional research is needed based on the majority vote (95.0\%;

\section{Endocrine-based treatment options for postmenopausal patients with HER2-negative metastatic breast cancer}

\author{
- Letrozole* + Palbociclib \\ - Fulvestrant + Palbociclib \\ - Letrozole*+ Ribociclib \\ - Letrozole /Anastrozole + Abemaciclib \\ - Fulvestrant + Abemaciclib \\ - Abemaciclib Monotherapy \\ - Exemestane + Everolimus \\ - Tamoxifen + Everolimus \\ - Letrozole + Everolimus \\ - Fulvestrant + Everolimus \\ - CDK4/6i beyond progression
}

\begin{tabular}{ccc}
\multicolumn{3}{c}{ Oxford } \\
LoE & GR & AGO \\
\hline 1b & B & ++ \\
1b & B & ++ \\
1b & B & ++ \\
1b & B & + \\
1b & B & + \\
3 & C & $+/-$ \\
1b & A & + \\
2b & B & + \\
2b & B & $+/-$ \\
2b & B & + \\
5 & D & -
\end{tabular}

*Data can also be extrapolated to other Als

Fig. 2. Endocrine-based therapeutic options for postmenopausal patients with HR+/HER2 - metastatic breast cancer. Prefinal slide according to AGO Mamma 2018 (version 2018 1E; www.ago-online.de)

abstain: 5.0\%) of the ABC4 panelists (LoE: 1D). The German experts also stress the urgent need for further selection criteria, e.g., biomarkers, molecular factors (including molecular imaging), or disease dynamics. From the German perspective, the point is also to identify those patients who are initially adequately treated with endocrine monotherapy and need no endocrine-based combination therapy in the first-line setting.

\section{Fulvestrant as a First-Line Option?}

The question whether fulvestrant is an adequate first-line option for postmenopausal patients with HR+/HER2- metastatic breast cancer who did not receive prior endocrine treatment and are unable to receive a CDK4/6 inhibitor in the first line (LoE: 1B) was withdrawn on site. There is therefore no voting result. The question is based on the data of the FALCON study [17]. Fulvestrant $(500 \mathrm{mg}$ ) achieved a longer median PFS in the first line compared to anastrozole, particularly in patients without visceral metastasis (LoE: 1B). However, there are so far no mature survival data. The survival results will be awaited and considered in the consensus manuscript. It was also emphasized that the optimal therapy sequence is currently still unknown.

The German experts see the monotherapy with fulvestrant as one option for first-line treatment of HR+/HER2- metastatic disease that may be sufficient for individual patients, particularly in case of no visceral involvement. Patients must, however, be informed that there is currently no confirmed selection criterion to identify these patients. Patients must also be informed about all available therapeutic options in the first-line situation, such as targeted endocrine-based therapy, and need to provide their informed consent to fulvestrant monotherapy against this background. 


\section{HR-Positive, HER2-Positive Advanced or Metastatic Breast Cancer}

\section{Endocrine Therapy + HER2 Blockade}

The ABC4 voting regarding HR+/HER2+ metastatic breast cancer started with the following initial statement: Patients with $\mathrm{HR}+$ / HER2+ metastatic breast cancer who receive endocrine therapy instead of chemotherapy belong to a highly selected patient group. Endocrine therapy should be combined with an anti-HER2 therapy (trastuzumab or lapatinib) since this combination induces a PFS advantage (and thus a longer time without chemotherapy) compared to endocrine therapy alone (LoE: 1A). The additional antiHER2 therapy did not show an overall survival benefit; however, no long-term follow-up was performed in the respective trials. Currently, the strategy of combined endocrine and anti-HER2 therapy is being compared to chemotherapy + anti-HER2 therapy in large controlled trials.

A clear majority of ABC4 panelists (80.4\%) voted to combine the endocrine therapy with dual HER2 blockade (trastuzumab/pertuzumab or trastuzumab/lapatinib) since this provides a longer PFS compared to single HER2 blockade. The decision for dual anti-HER2 therapy has to be made in consideration of potentially more side effects, higher costs, and the lack of an overall survival benefit.

From the German perspective, it needs to be added that dual anti-HER2 blockade in combination with single-agent taxane therapy is currently the standard first-line treatment for HR+/HER2+ metastatic breast cancer $[5,6]$ because it provides a substantial overall survival benefit. Contraindications against taxane chemotherapy, such as 'paclitaxel weekly' as favored in Germany, are very rare. If a patient refuses chemotherapy, she must be informed that no survival benefit was shown for the endocrine combination. The German experts recommend including patients with HR+/HER2+ metastatic breast cancer in the randomized DETECT V (CHEVENDO) trial, where patients receive dual HER2 blockade (trastuzumab/pertuzumab) in combination with endocrine or chemotherapy. DETECT $\mathrm{V}$ also focuses on the patient quality of life in both study arms.

\section{Anti-HER2 Therapy and Maintenance Therapy}

Endocrine maintenance therapy in combination with continued HER2 blockade is a reasonable therapeutic strategy after chemotherapy for patients with $\mathrm{HR}+/ \mathrm{HER} 2+$ metastatic breast cancer who successfully received chemotherapy + anti-HER2 therapy even in the absence of randomized study data; this was the majority vote by the $\mathrm{ABC} 4$ panelists ( $80.4 \%$; abstained: $17.0 \%$ ). The optimal duration of maintenance therapy should be validated in clinical studies. Currently valid: Maintenance therapy should only be discontinued in case of disease progression, unacceptable side effects, or patient request. - The German experts agree with all of this.

\section{Triple-Negative Advanced or Metastatic Breast Cancer}

\section{Relevance of the Androgen Receptor}

Patients with advanced triple-negative breast cancer (TNBC) and positive (overexpressed) androgen receptor $(\mathrm{AR}+)$ who no longer respond to the available standard treatments have a particularly poor prognosis. In light of this, the $\mathrm{ABC} 4$ panelists discussed whether the AR inhibitor bicalutamide (150 mg/day) is an option for these patients in individual cases. The relevance of AR inhibition in AR+ metastatic TNBC has not yet been validated in randomized phase III trials.

In a statement modified on site, the $\mathrm{ABC} 4$ panelists suggested the $\mathrm{AR}$ in a majority vote (85.3\%; abstained: $14.6 \%)$ as a potential target in AR+ TNBC, but then emphasized that there is no standardized method of assessment. Limited data suggest some efficacy of the AR inhibitors bicalutamide and enzalutamide (second generation). However, further study data are needed, which is why AR inhibitors should not be used routinely. The ABC4 panelists pleaded for increased research activities and clinical trials.

From the German perspective, bicalutamide can be a therapeutic option for an adequately informed individual patient with AR+ metastatic TNBC when the standard therapy options have been exhausted. The German experts add that a predictive AR test is currently not available. They confirm that the immunohistochemical testing of AR positivity is not validated. They add that the development of enzalutamide for breast cancer treatment was stopped and that, consequently, further study data on AR inhibition in breast cancer cannot be expected anytime soon.

\section{BRCA-Associated Advanced or Metastatic Breast Cancer}

\section{Early Genetic Testing}

For patients with advanced or metastatic breast cancer and positive family history, all ABC4 panelists (100\%!) recommended early genetic testing since the test result could affect treatment decisions. The ABC4 panelists demanded to include the recommended genetic testing of these patients into international and national guidelines (LoE: expert opinion).

The German experts agree with both statements in reference to the German guidelines [5-7]: In Germany, the current recommendation is to perform genetic testing in patients with a family history and in those with TNBC (no age restriction and no positive family history required), as well as in very young patients ( $<35$ years, also in those without a positive family history). If necessary, patients fulfilling the above criteria should be referred to a specialized center or a geneticist for testing. Therapeutic options according to the current AGO recommendations can be seen in figure $3[5,6]$.

\section{Only BRCA1/2 Germline Testing Validated to Date}

There was also consensus (100\%!) among the ABC4 panelists that genetic testing should be performed based on a positive family 


\section{Therapy of BRCA1/2-associated Breast} Cancer+

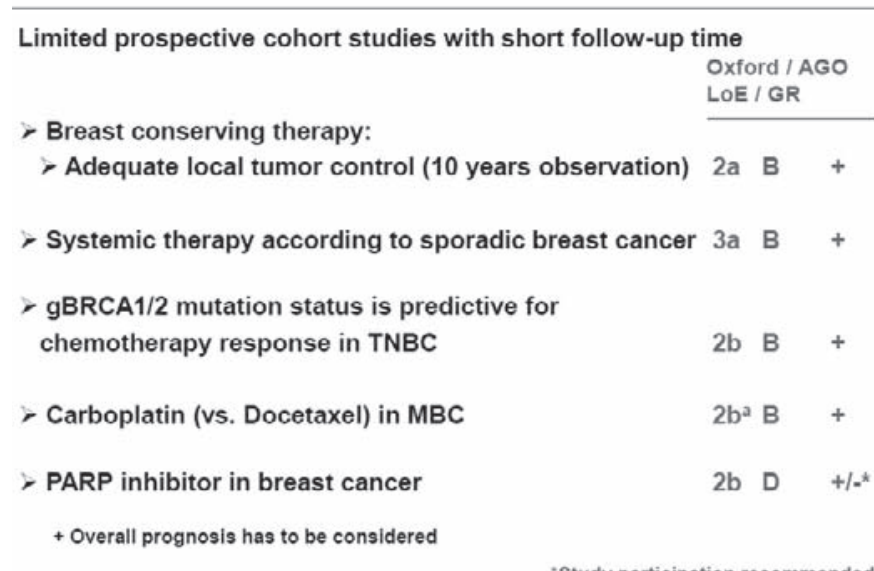

Fig. 3. Therapeutic options in $B R C A 1 / 2$-associated breast cancer according to the AGO Mamma 2017; modified according to $[5,6]$.

history and that currently only $B R C A 1 / 2$ germline mutations have an evidence-based therapeutic impact and thus potential clinical benefit in this context. Testing for other moderate- to high-risk genes can be considered after consulting the geneticist/genetic counselor. In this case, the patients must be informed that the test result currently does not immediately affect the treatment but could benefit family members (LoE: expert opinion).

In another statement, most of the $\mathrm{ABC} 4$ panelists agreed (82.5\%; abstain: $15.0 \%)$ that somatic BRCA1/2 mutation testing should currently not be applied in clinical routine. Therapeutic consequences potentially resulting from somatic testing must further be evaluated under research conditions (LoE: expert opinion).

The German experts also agree with these voting results. The German experts confirm that the BRCA determination in the tumor tissue currently has no therapeutic consequences in breast cancer. However, they point out that not all tumor mutations may be detected by germline testing [18]. The clinical relevance of tumor tissue testing should therefore be further studied, preferentially in the context of controlled clinical trials.

\section{Impact of Poly(ADP-Ribose) Polymerase Inhibition}

The poly(ADP-ribose) polymerase (PARP) inhibitor olaparib is currently not approved in advanced or metastatic hereditary breast cancer. However, the majority of the $\mathrm{ABC} 4$ panelists $(80.0 \%$; abstained: $17.5 \%$ ) considered olaparib as a reasonable therapeutic option for patients with advanced or metastatic BRCA-associated TNBC or luminal-like breast cancer - in the latter case, only if endocrine therapy is not an option anymore. Patients should have received prior (neo-)adjuvant or first-line treatment anthracyclines \pm taxanes and should not be resistant to platinum salts.

The ABC4 panelists based their vote on the phase III study OlympiAD demonstrating that olaparib offers the chance for a longer PFS with a better side effect profile and simultaneous improvement in quality of life compared to chemotherapy of physician's choice in patients with a germline $B R C A$ mutation. Overall survival results are not yet mature. It is also unclear how PARP inhibitors perform in comparison to platinum complexes (LoE: 1A). Patients for whom olaparib may represent a therapeutic option need to be specified, based on the discussion of the ABC4 panelists on site.

The German experts basically agree. They stress once more that olaparib is currently not approved in advanced or metastatic breast cancer and therefore can only be considered as an option in individual patients (off-label application in agreement with health insurance company may be necessary).

\section{Biosimilars}

The ABC4 panelists strongly recommended the use of biosimilars in breast cancer as treatment (e.g. trastuzumab) as well as supportive care (e.g. growth factors) with a clear majority (90.2\%; abstained: 7.3\%). The condition for the use of a biosimilar is that it is approved and has successfully undergone the stringent development and validation process required by the European Medicines Agency (EMA), The US Food and Drug Administration (FDA), or a comparable agency (LoE: 1A). - The German experts agree. They point out that the first biosimilars for breast cancer therapy have already been approved since November 2017.

\section{Precision Medicine}

\section{Multigene Panels in Focus}

The relevance of multigene test procedures, for example nextgeneration sequencing (NGS), or other technologies using tumor DNA for clinical routine use has already been discussed for several years. The rationale behind this is the hope for targeted patient selection or personalized therapy with better patient outcome. A clear majority of the ABC4 panelists (82.9\%) currently does not see multigene testing as an option for clinical routine. The experts pointed out that, so far, controlled clinical trials have not shown any advantages for patients, thus leaving it unclear whether the outcome can be favorably affected.

Regardless of this, according to the ABC4 panelists, NGS is already used in prospective molecular decision studies to select patients for corresponding therapy trials. This requires that patients agree to participate in a clinical study evaluating such new diagnostic and therapeutic options, and to subsequently receive treatment at a center participating in clinical trials or registries with experience in using off-label drugs. Tests that were specifically developed and validated for the use of certain drugs will play a role in clinical routine as soon as such drugs have been officially approved according to the majority vote of the $\mathrm{ABC} 4$ panelists (LoE: 1D).

The German experts agree with these statements. So far, there are no such registries in Germany with corresponding patient data. In Germany, multigene testing should thus preferably be conducted at 'Comprehensive Cancer Centers' (CCCs) that have a molecular tumor board including gyneco-oncologists. This will facili- 
tate the setup of corresponding databases and bio-banks. The benefit of such clinical and translational registries is already becoming apparent in the ongoing PRAEGNANT study in Germany.

\section{Analyze Circulating Cell-Free Tumor DNA?}

The analysis of circulating tumor DNA (ctDNA) is not an option in clinical routine and is not recommended by the $\mathrm{ABC} 4$ panelists in order to detect disease progression or to select specific treatments (74.3\%; abstain: 10.2\%; insufficient data: 15.3\%) (LoE: 1D). The German experts agree and point out that the clinical impact of ctDNA as well as that of circulating tumor cell (CTC) analyses are currently being evaluated in Germany in the context of translational research projects and large clinical trials (e.g. DETECT, PRAEGNANT).

\section{Microsatellite Instability in Breast Cancer}

The vote by the ABC4 panelists on the relevance and consequences of high-frequency microsatellite instability/mismatch repair deficiency (MSI-H/MMRD) was controversial: About $40 \%$ of the ABC4 panelists (41.4\%) thought that programmed death-1 (PD1)-directed inhibitors are a possible option for these patients (LoE: expert opinion). Just about half of the ABC4 panelists (48.7\%) did not agree due to insufficient data. About 10\% (9.7\%) abstained.

The German experts refer to the Amsterdam II criteria which include clinical criteria comprising personal or family history suggesting potential MSI positivity [19]. These criteria may need to be systematically included in each patient's medical history. The Amsterdam criteria are considered a prerequisite for MSI testing. From the German perspective, anti-PD1-directed therapy with a PD1 antibody such as pembrolizumab should be supported in case of a positive MSI test result based on the recent FDA approval and the limited treatment options for such patients [20].

\section{NTRK Fusion in Breast Cancer}

A simple majority of the ABC4 panelists (47.3\%; abstain: 23.6\%) did not think that there are sufficient data for use of a specific tyrosine receptor kinase (TRK) inhibitor (entrectinib) in patients with advanced or metastatic breast cancer and NTRK gene fusion (NTRK = neurotrophic tyrosine receptor kinase). Less than onethird of the ABC4 panelists (28.9\%) would possibly consider use of a TRK inhibitor on these patients. - From the German perspective, NTRK fusion currently does not play a role in clinical routine. Furthermore, the incidence is very low. The discussion of this topic at the $\mathrm{ABC} 4$ reflects a future development where molecular tumor boards are gaining importance. This again emphasizes the need to set up tumor registries with the corresponding tumor data in Germany in order to advance precision medicine for increasingly individualized therapy decisions.

\section{Immunotherapy in Breast Cancer}

Immunotherapy is already an important new pillar in the treatment of several solid tumors. However, so far, there are no reliable validated data for breast cancer. More than $80 \%$ of the ABC4 pan- elists (84.6\%) thus do not think that immunotherapy is indicated in advanced or metastatic breast cancer outside of clinical trials. Regardless of the biological subtype, immunotherapy should not be applied or recommended in clinical routine. The ABC4 panelists referred to ongoing clinical trials evaluating the relevance of immunotherapy in the diverse breast cancer subtypes (LoE: expert opinion).

The German experts basically agree with the majority vote by the ABC4 panelists. However, they could not rule out that immunotherapy may be an option in individual patients after exhaustion of the available standard therapies and if the patient's general condition and life expectancy support this decision. It should be noted that severe and new toxicities are rare but possible during immunotherapy, which is why significant oncological expertise is needed. The German experts add that there is currently no validated predictive biomarker for the use of immunotherapy in breast cancer. They also emphasize that there is currently no definitive evidence in breast cancer. First phase-III study results are expected by 2018 .

\section{Treatment of Brain Metastases}

Radionecrosis after stereotactic radiation of brain metastases is a rare complication that can develop years after radiation. The risk increases with the increasingly longer survival time of metastatic breast cancer patients, particularly after repeated irradiation of the brain. Differential diagnosis - demarcation due to tumor progression - can be difficult. According to the majority vote by the ABC4 panelists (60.5\%; abstain: $31.5 \%)$, high-dose steroids are the treatment of choice for symptomatic patients with radionecrosis. If the patients do not adequately respond to this, the ABC4 panelists see an option in treatment with bevacizumab $(7.5 \mathrm{mg} / \mathrm{kg}$ every 2 weeks) to lower the elevated intracranial pressure in the surrounding tissue. The patients should receive an average of 4 cycles of bevacizumab. Prospective randomized studies are necessary to evaluate further therapeutic options for these patients (LoE: IIIB). - The German experts add that radionecrosis is a rare clinical diagnosis and that treatment of these patients should be discussed in the interdisciplinary tumor board.

\section{Brain Metastases in HER2 + Metastatic Breast Cancer}

If there are no more local treatment possibilities for patients with metastatic HER2+ breast cancer and progressive brain metastasis, or if these are no longer effective and the brain is the primary metastasis site, a clear majority of the ABC4 panelists (85.0\%) considered systemic treatment as a reasonable option (LoE: IIIA).

The ABC4 panelists additionally referred to earlier statements that are still valid: i) Systemic therapy should not be changed in patients who develop brain metastases if extracranial disease is controlled and the brain metastases can be treated by local therapy (LoE: 1C). ii) If brain metastases are the only metastatic site in patients with HER2+ breast cancer, there is no evidence that chemotherapy applied in addition to local treatment impacts disease progression. 
However, it is recommended to continue the anti-HER2 therapy (trastuzumab) if it was discontinued in the meantime (LoE: 1C).

The German experts agree with the new statement and refer to data that prove an effect on progressing brain metastases, particularly for trastuzumab-emtansin (T-DM1) and lapatinib [21-24]. The treatment of brain metastases must be discussed in an interdisciplinary tumor board. Valuable data is expected from the German Breast Group (GBG) registry on brain metastases in breast cancer, initiated and led by the Department of Obstetrics and Gynecology of the University of Hamburg (Prof. Volkmar Müller, PD Dr. Isabell Witzel).

\section{Important Definitions}

\section{Adequate OFS}

The discussion during the ABC4 consensus on what adequate OFS should look like in premenopausal patients with advanced or metastatic breast cancer was controversial at times. Therefore, the respective statement for the $\mathrm{ABC} 4$ consensus publication will be discussed again later and revised, if necessary.

Nevertheless, a majority of the ABC4 panelists $(84.2 \%$; abstained: $10.5 \%)$ agreed with the statement suggested on site: Adequate OFS can be achieved in premenopausal patients by continuous administration of a luteinizing hormone-releasing hormone (LHRH) agonist, by bilateral ovariectomy or by pelvic radiation of the ovaries (ovarian ablation). The $\mathrm{ABC} 4$ panelists stated that pelvic radiation is the least preferable option. For an optimal result, OFS using a gonadotropin-releasing hormone ( $\mathrm{GnRH})$ agonist should be performed every 28 days in premenopausal women. Regular checks of the follicle-stimulating hormone (FSH) and estradiol levels are recommended in the beginning, even in young patients with amenorrhea after prior cancer therapy.

The ABC4 panelists pointed out that all endocrine measures in the young patients with endocrine-sensitive advanced or metastatic breast cancer require long-term OFS. In the decision as to which OFS method is preferable, patient wishes regarding fertility preservation, patient adherence to monthly injections over a long period of time, as well as therapy costs and potential surgical alternatives should be weighed against one another.

The German experts emphasize that regular follow-up examinations are particularly important in the beginning of a GnRH agonist therapy (every 28 days). This is particularly important during combined administration with an AI. In this case, the German experts recommend regular measurements of the hormone status (i.e. FSH and estradiol levels) for the first 3-6 months. According to the guidelines $[5,6]$, long-term ovarian function suppression is necessary in premenopausal patients during endocrine-based therapy in advanced or metastatic breast cancer. Bilateral salpingo-oophorectomy (BSO) should be discussed with these patients since in metastatic disease ovarian function suppression is practically permanent. The German experts add that the patient must be informed about the need for reliable contraception during systemic OFS (reference to statement on preserving fertility) [8].

\section{Update on Locally Advanced Breast Cancer}

\section{Duration of Adjuvant HER2 Blockade}

The ABC4 panelists defined locally advanced breast cancer (LABC) as inoperable, locally advanced breast cancer without distant metastases. A clear majority of the ABC4 panelists (84.6\%; abstained: $15.3 \%$ ) agreed on the duration of adjuvant anti-HER2 therapy in patients with inflammatory or non-inflammatory HER2+ LABC, with the following statement: If these patients are in complete remission after adequate neoadjuvant systemic therapy and subsequent locoregional treatment, the optimal duration of adjuvant anti-HER2 therapy is unclear. The ABC4 panelists therefore recommended defining the adjuvant therapy duration in dependence on tolerability of the treatment, logistical efforts, and costs. Patients being treated with a curative intent should receive adjuvant anti-HER2 therapy for the total of 1 year (LoE: expert opinion).

The German experts emphasize, in reference to the current AGO recommendations $[5,6]$, that adjuvant anti-HER2 therapy is limited to 1 year in case of a curative treatment intent. Given the APHINITY trial results [25] with the benefit of the dual blockade particularly in patients at high risk of recurrence (e.g., positive nodal status and negative steroid hormone receptor status), adjuvant application of dual HER2 blockade may be discussed as an option in patients with LABC. After 1 year of trastuzumab therapy, additional subsequent anti-HER2 therapy with neratinib could be considered in HR+/HER2+ breast cancer [26].

\section{Inflammatory $L A B C$}

There was no vote during the $\mathrm{ABC} 4$ meeting on the following 2 statements on inflammatory LABC due to controversial discussions:

- There was a controversial discussion whether breast-preserving surgery could be considered in individual cases of inflammatory LABC if the primary tumor is confined and complete regression of the affected skin areas was achieved after systemic therapy based on clinical examination and imaging (LoE: IIIB).

- The statement that sentinel lymph node biopsy (SLNB) is not an option in inflammatory LABC due to a potentially high rate of incorrect negative findings was also controversially discussed. The high false-negative rate may be due to lymphovascular tumor-related emboli in the parenchyma of breast and skin which may prevent normal lymph flow into the axilla, making evaluation of the lymph nodes ('lymphatic mapping') by imaging more difficult (LoE: IIID).

The German experts point out that neoadjuvant treatment followed by modified radical mastectomy (i.e. including axillary dissection) and radiotherapy (chest wall and regional lymph nodes) is still standard in inflammatory LABC $[5,6]$.

\section{SLNB in Inoperable LABC}

A still valid $A B C$ consensus statement from a few years ago indicates that most patients with $\mathrm{LABC}$ who were primarily inoperable are able to receive secondary surgery \pm radiation after effective neoadjuvant systemic treatment. These patients generally get a 
modified radical mastectomy. However, breast-conserving surgery with axillary dissection may be considered in individual cases with excellent response to the preoperative treatment (LoE: 2B).

The ABC4 panelists agreed on the following new statement: If patients with a low tumor load in the axilla or a clinically normal axilla ( $\mathrm{cN} 0-\mathrm{cN} 1)$ upon initial diagnosis achieve clinical complete remission ( $y c N 0$ ) during neoadjuvant systemic therapy, an SLNB may be an option. However, the requirement is that this is performed according to the guidelines and recommendations for SLNB after primary systemic therapy. This includes marking with both blue dye and technetium, clipping or rather marking of suspicious lymph nodes, and removal of at least 3 sentinel lymph nodes during surgery after neoadjuvant therapy (LoE: IIIA). Two-thirds of the ABC4 panelists (62.1\%; abstained: $21.6 \%)$ agreed with this statement.

The German experts do not agree with this statement. The German experts refer to the AGO recommendations according to which axillary dissection is the standard axilla treatment in primarily LABC that becomes operable after neoadjuvant therapy $[5,6]$.

\section{General Statements}

\section{Almost no Changes Regarding Chemotherapy}

Chemotherapy is a central pillar in the treatment of LABC or metastatic breast cancer. There was only 1 modified statement on metronomic chemotherapy during the $2017 \mathrm{ABC} 4$ consensus meeting. Beside this, all respective statements of the previous $\mathrm{ABC}$ consensus conferences are still valid. - The German expert group additionally points to the current AGO recommendations [5] as well as the most recent $S 3$ guideline on the treatment of breast cancer [7].

From the German perspective, metronomic chemotherapy is a reasonable approach for patients with metastatic breast cancer [5, 6]. The prepared statement was not voted on in the ABC4 meeting since a data update is expected shortly.

\section{Preserving Fertility in Advanced and Metastatic Breast Cancer}

The German expert group agrees with the ABC4 statement that the question of fertility must be addressed with patients of childbearing age (and their partners) before the start of treatment and must be discussed in the context of the LABC or the non-curable metastatic disease setting. All ABC4 panelists (100\%!) agreed with this and argued that the discussion with the patient must also include information on their prognosis and the potential impact of a pregnancy, such as potential interruption of ongoing treatment (LoE: expert opinion). The German experts add that particularly the reduced life expectancy and the negative impact of a therapy interruption on this already reduced life expectancy must also be addressed.

\section{Integrative Medicine}

According to the current general statement of the ABC4 consensus, complementary and integrative medicine (CIM) means the use of potentially complementary therapies together with conven- tional therapies (LoE: expert opinion). The published consensus manuscript will contain further statements on integrative medicine'. There was no on-site vote on 'integrative medicine' for reasons of time. From the German perspective, 'integrative medicine' describes the utilization of complementary methods in addition to standard therapy. For further information, the German experts refer - in addition to the AGO recommendations $[5,6]$ - to the 'Onkopedia Guideline Complementary and Alternative Therapies' [27] and the 'Colloquium Senologie 2017/2018' [28].

\section{Supportive and Palliative Treatment}

For reasons of time, there was also no vote at the ABC4 congress on questions of supportive therapy and palliative treatment of patients with advanced or metastatic breast cancer. Consequently, there is no comment from the German experts and the published manuscript needs to be awaited. The focus will be on the management of chemotherapy-induced peripheral neuropathy (CIPN), hand-foot syndrome (HFS), and mucositis and stomatitis. - The German expert group refers to the current recommendations by the AGO Mamma $[5,6]$ and the respective publication in 'Colloquium Senologie 2017/2018' [28].

\section{ABC4 - Statements by Patient Advocates}

The ABC conference deliberately and specifically promotes communication with patient advocates and additional representatives of the medical profession. As in the past, numerous patient advocates from several countries participated in the ABC4 conference. Altogether, 82 representatives from Europe, Asia, the Middle East, Africa, Australia, and North, South, and Central America were present. The patient advocates summarized the results of their work in 4 core statements, which were presented during the consensus session of the $\mathrm{ABC} 4$ conference and which were emphasized by the Australian panelist and patient advocate Danielle Spence (Breast Cancer Network) in her lecture.

The patient advocates call for:

- Every patient with advanced breast cancer should have access to the most recent therapeutic options as well as specific new treatment at the respective certified centers.

- Every patient with advanced breast cancer should be treated by a specialized multidisciplinary team at specialized breast centers or institutions cooperating with these certified centers. This includes specialized side effect management and a nursing team specialized in metastatic breast cancer.

- Every patient with advanced breast cancer should be informed early - if possible already in an early disease setting - of survivorship issues. Also palliative care should be addressed and offered at an early stage.

- A quality assurance program must be implemented at each specialized center in order to ensure that the patient receives quality-assured treatment and care at every stage during her disease 
- from screening to diagnosis and therapy to rehabilitative measures, follow-up care, and palliative therapy offers.

On behalf of all patient advocates, Spence expressed her thanks for the possibility of participating in the ABC4 conference and the international exchange among the advocates as well as with the physicians.

The German expert group supports the demands by the patient advocates and their resulting statements. Renate Haidinger, Doris Schmitt, and Eva Schumacher-Wulf were on site, representing patients and breast cancer advocates from Germany. From the German perspective, a central demand is the interdisciplinary cooperation at specialized centers. In addition to quality-assured oncological treatment, this includes the management of side effects as well as adequate palliative medical and supportive therapeutic choices that need to be explained to the patient.

The so-called 'Global Alliance' with substantial German participation was founded after the ABC4 conference to coordinate worldwide measures for advanced and metastatic breast cancer. The 'Global Alliance' defined the following goals for the next 2 years:

- Goal 1: Double the median overall survival for patients with advanced and metastatic breast cancer to at least 4 years by 2025 .

- Goal 2: Improve the quality of life for advanced and metastatic breast cancer patients in clinical practice.

- Goal 3: Increase the availability of robust epidemiology and outcomes data for advanced and metastatic breast cancer.

- Goal 4: Increase the availability of and access to multidisciplinary care, including palliative, supportive, and psychosocial assistance for patients, families, and caregivers to ensure that patients are receiving the best treatment experience from health insurance companies.

\section{Conclusion and Outlook}

The ABC4 conference again offered an informative platform for important discussions on the most recent developments in advanced and metastatic breast cancer. The $\mathrm{ABC}$ consensus achieved an important contribution in terms of standardizing treatment for advanced and metastatic breast cancer on an international level and optimizing treatment worldwide. In this respect, the $\mathrm{ABC}$ congress is a valuable addition to the St. Gallen consensus conference on early breast cancer. The next $\mathrm{ABC} 5$ consensus conference will take place on November 14-16, 2019.

\section{Acknowledgements}

The meeting of the German experts in Lisbon was supported and organized by the educational company AURIKAMED Institut GmbH. The authors would like to thank Birgit-Kristin Pohlmann, Nordkirchen, for her editorial assistance. The authors are solely responsible for the final content of the manuscript.

\section{Disclosure Statement}

N.H. received honoraria from Amgen, Celgene, Novartis, Pfizer, Roche, Sandoz. D.L. received honoraria from Celgene, Pfizer, Novartis, Roche, Eisai, AstraZeneca, Amgen, MSD, Pierre Fabre, and L'Oreal. N.M., M.U., D.A., and S.B. have no conflict of interest. J.E. received honoraria from Celgene, Eisai, GSK, Janssen, Lilly, Novartis, Pfizer, Roche, a research grant from Celgene and a travel grant from Celgene, GSK, Novartis, Pfizer, Roche. R.H. and L.M. have no conflict of interest. V.M. received honoraria from Amgen, AstraZeneca, Celgene, Daiichi-Sankyo, Eisai, Pfizer, Pierre-Fabre, Nektar, Novartis, Roche, TESARO, Teva, Janssen-Cilag. E.R. received honoraria from Amgen, AstraZeneca, Celgene, Janssen-Cilag, Novartis, Pierre Fabre, Roche. R.W. received honoraria from Celgene, Pfizer, Novartis, Roche, Eisai, AstraZeneca, Amgen, MSD, Pierre Fabre. C.T. received honoraria from Amgen, AstraZeneca, Celgene, Lilly, Novartis, NanoString, Pfizer, Puma, Roche.

\section{References}

1 Cardoso F, Costa A, Norton L, et al: 1st International consensus guidelines for advanced breast cancer (ABC 1). Breast 2012;21:242-252.

2 Cardoso F, Costa A, Norton L, et al: ESO-ESMO 2nd international consensus guidelines for advanced breast cancer (ABC2). Breast 2014;23:489-502.

3 Cardoso F, Costa A, Senkus E, et al: ESO-ESMO 3rd international consensus guidelines for advanced breast cancer (ABC3). Breast 2017;31:244-259.

4 www.esmo.org/Guidelines/ESMO-Guidelines-Methodology (last accessed November 13, 2017).

5 Liedtke C, Thill M, Jackisch C, Thomssen C, Müller V, Janni W; on behalf of the AGO Breast Committee: AGO recommendations for the diagnosis and treatment of patients with early breast cancer: update 2017. Breast Care 2017;12:172-183.

6 Thill M, Liedtke C, Solomayer E-F, Müller V, Janni W, Schmidt M; on behalf of the AGO Breast Committee: AGO recommendations for the diagnosis and treatment of patients with advanced and metastatic breast cancer: update 2017. Breast Care 2017;12:184-191.
7 www.awmf.org (retrieved: 11/13/2017), www.leitlinienprogramm-onkologie.de, consultation version S3 guideline 'Early detection, diagnostics, therapy and aftercare of breast cancer', long version 0.4.1., August 2017, AWMF registry no. 032-045OL.

${ }_{8}$ Paluch-Shimon S, Pagani O, Partridge AH, et al: ESOESMO 3rd international consensus guidelines for breast cancer in young women (BCY3). Breast 2017;35: 203-217.

9 Baselga J, Campone M, Piccart M, et al: Everolimus in postmenopausal hormone-receptor-positive advanced breast cancer. N Engl J Med 2012;366:520-529.

10 Beck JT, Hortobagyi GN, Campone M, et al: Everolimus plus exemestane as first-line therapy in HR+, HER2- advanced breast cancer in BOLERO-2. Breast Cancer Res Treat 2014;143:459-467.

11 Kornblum NS, Manola J, Klein P, et al: PrECOG 0102: a randomized, double-blind phase II trial of fulvestrant plus everolimus or placebo in post-menopausal women with hormone-receptor positive, HER2-negative metastatic breast cancer resistant to aromatase inhibitor therapy. SABCS 2016;abstr S1-02.
12 Finn RS, Martin M, Rugo HS, et al: Palbociclib and letrozole in advanced breast cancer. N Engl J Med 2016;375:1925-1936.

13 Hortobagyi GN, Stemmer SM, Burris HA, et al: Ribociclib as first-line-therapy for HR-positive, advanced breast cancer. N Engl J Med 2016;375:1738-1748.

14 Hortobagyi GN: Updated results from MONALEESA-2, a phase 3 trial of first-line ribociclib + letrozole in hormone receptor-positive, HER2-negative advanced breast cancer. J Clin Oncol 2017;35(15S):1038.

15 Christofanilli M, Turner NC, Bonarenko I, et al: Fulvestrant plus palbiciclib versus fulvestrant plus placebo for treatment of hormone-receptor-positive, HER2negative metastasic breast cancer that progressed on previous endocrine therapy (PALOMA-3): final analysis of the multicentre, double-blind, phase 3 randomised controlled trial. Lancet Oncol 2016;17:425439.

16 Harbeck N, Iyer S, Turner N, et al: Quality of life with palbociclib plus fulvestrant in previously treated hormone receptor-positive, HER2-negative metastatic breast cancer: patient-reported outcomes from the PALOMA-3 trial. Ann Oncol 2016;27:1047-1054. 
17 Robertson JF, Bondarenko IM, Trishkina E, et al: Fulvestrant $500 \mathrm{mg}$ versus anastrozole $1 \mathrm{mg}$ for hormonereceptor-positive advanced breast cancer (FALCON): an international, randomised, double-blind phase 3 trial. Lancet 2017;388:2997-3005.

18 Hahnen E, Lederer B, Hauke J, et al: Germline mutation status, pathological complete response, and disease-free survival in triple-negative breast cancer: secondary analysis of the GeparSixto randomized clinical trial. JAMA Oncol 2017;3:1378-1385.

19 Vasen HF, Watson P, Mecklin JP, Lynch HT: New clinical criteria for hereditary nonpolyposis colorectal cancer (HNPCC, Lynch syndrome) proposed by the International Collaborative Group on HNPCC. Gastroenterology 1999;116:1453-1456.

20 www.fda.gov/NewsEvents (last accessed December 2017).
Bartsch R, Berghoff A, Pluschnig U, et al: Impact of anti-HER2 therapy on overall survival in HER2-overexpressing breast cancer patients with brain metastases. Br J Cancer 2012;106:25-31

22 Bartsch R, Berghoff AS, Preusser M: Breast cancer brain metastases responding to primary systemic therapy with T-DM1. J Neurooncol 2014;116:205-206.

23 Bachelot T, Romieu G, Campone M, et al: Lapatinib plus capecitabine in patients with previously untreated brain metastases from HER2-positive metastatic breast cancer (LANDSCAPE): a single-group phase 2 study. Lancet Oncol 2013;14:64-71.

24 Montemurro F, Ellis P, Delaloge S, et al: Safety and efficacy of trastuzumab emtansin in 399 patients with central nervous system metastases: exploratory subgroup analysis from the KAMILLA study. SABCS 2016;abstr P1-12-10.
25 Von Minckwitz G, Procter M, de Azambuja E, et al: Adjuvant pertuzumab and trastuzumab in early HER2positive breast cancer. N Engl J Med 2017;377:122131.

26 Martin M, Holmes FA, Ejletersen B, et al: Neratinib after trastuzumab-based adjuvant therapy in HER2positive breast cancer (ExteNET): 5-year analysis of a randomised, double-blind, placebo-controlled phase 3 trial. Lancet Oncol 2017;18:1688-1700.

27 www.oncopedia.com/de/news/komplementaere-undalternative-therapieverfahren (last accessed November, 2017).

28 Untch M, Harbeck N, Thomssen C: Colloquium Senologie 2017/2018, ed 1. München, Lukon, 2017.

29 Dykewicz CA: Summary of the guidelines for preventing opportunistic infections among hematopoietic stem cell transplant recipients. Clin Infect Dis 2001;33:139-144. 